\title{
Discordant tumor response in the treatment of ALK-rearranged non-small cell lung cancer leads to the diagnosis of synchronous multiple primary lung cancer
}

\author{
Benny Johnson ${ }^{1^{*}}$, Maged Khalil ${ }^{2}$ \\ ${ }^{1}$ Department of General Internal Medicine, Geisinger Medical Center, Geisinger Health System, Danville, USA; \\ *Corresponding Author: benjohnson2010@gmail.com \\ ${ }^{2}$ Department of Hematology-Oncology, Geisinger Medical Center, Geisinger Health System, Danville, USA
}

Received 24 January 2013; revised 27 February 2013; accepted 3 March 2013

\begin{abstract}
The advent of targeted molecular therapy against the EML4-ALK fusion gene is the latest therapeutic intervention for a subset of patients with non-small cell lung cancer (NSCLC). Crizotinib (Xalkori) is an orally available small molecule tyrosine kinase inhibitor proven in clinical trials to significantly impact progression free survival and overall response rate. We present a case of a 56-year-old male with NSCLC whose lack of a positive treatment response to this therapy led to the clinical suspicion and identification of the underdiagnosed entity known as synchronous multiple primary lung cancer (SMPLC).
\end{abstract}

Keywords: Non-Small Cell Lung Cancer; Crizotinib; Synchronous Multiple Primary Lung Cancer;

EML4-ALK Fusion Gene

\section{INTRODUCTION}

Lung Cancer is the leading cause of cancer related mortality in the United States and worldwide [1]. In nonsmall cell lung cancer (NSCLC), EML 4-ALK is a fusion gene responsible for encoding a cytoplasmic chimeric protein with constitutive kinase activity [2]. Crizotinib (Xalkori) is an orally available small molecule mesenchymal-epithelial transition/ALK multi-targeted receptor kinase inhibitor proven in early phase clinical trials to impact disease control rates in NSCLC.

\section{CASE REPORT}

A 56-year-old Caucasian male with a sixty pack year tobacco abuse history presents to the clinic with complaints of two weeks of generalized malaise and new onset dyspnea on exertion. He denies fevers, chills, productive cough, swollen glands, night sweats, or weight loss. A chest $\mathrm{x}$-ray revealed multiple bilateral pulmonary nodules with a right upper lobe mass highly concerning for malignancy. A computed tomography (CT) scan of the chest abdomen and pelvis was performed and upon initial review of the film the patient was sent to the emergency department as a right pulmonary embolus, large pericardial effusion, left renal vein thrombosis, and right upper lobe mass with multiple pulmonary nodules was diagnosed.

Upon evaluation, the patient was afebrile with a systolic blood pressure of 90/60 and heart rate of 120. Cardiac examination revealed sinus tachycardia and findings consistent with cardiac tamponade. The remainder of the physical exam was unremarkable. An electrocardiogram revealed electrical alternans. A bedside echocardiogram revealed a massive pericardial effusion. Emergent pericardiocentesis was performed and $1800 \mathrm{ml}$ of hemorrhagic fluid drained. IVC filter placement, pericardial window with chest tube insertion, and nodule biopsy was performed by Thoracic surgery. Fluid cytology along with pathology from the lung nodules and pericardium was

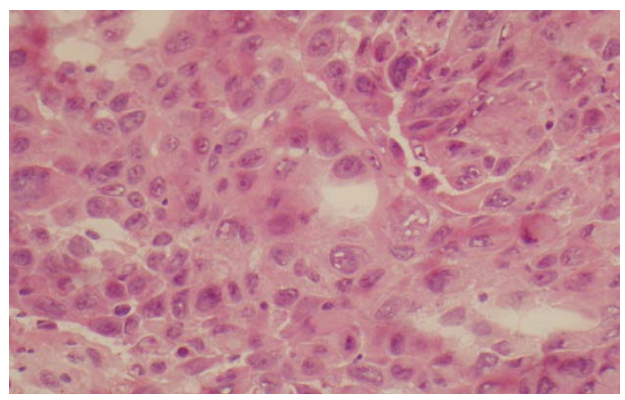

Figure 1. Fluid cytology along with pathology from the lung nodules and pericardium was positive for malignant pulmonary adenocarcinoma. 
positive for malignant pulmonary adenocarcinoma (Figure 1). The patient was referred to Oncology for further evaluation and treatment. PET CT of chest, abdomen and pelvis revealed multiple FDG avid nodules in the right

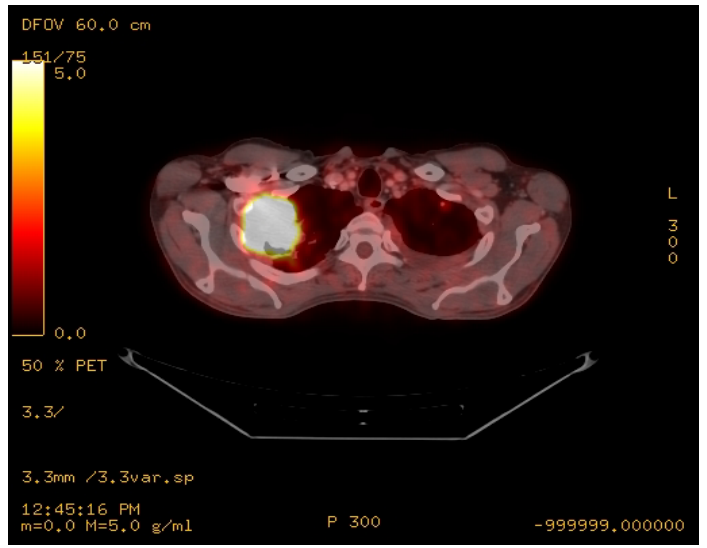

(a)
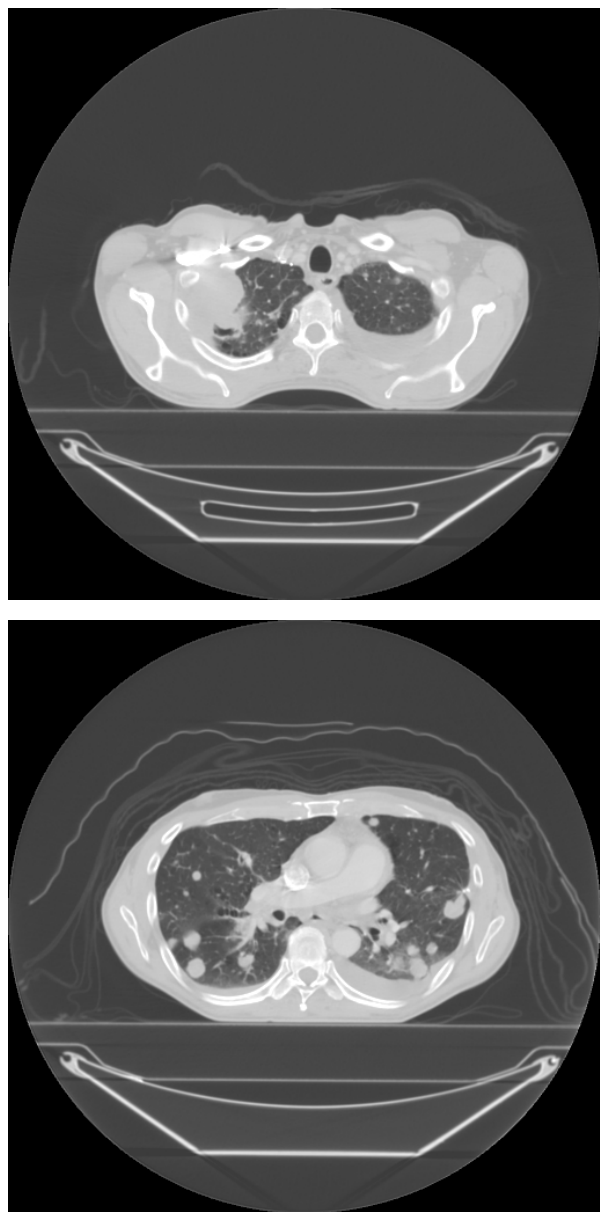

and left lung with a large intensely FDG avid mass in the right upper lobe, significant cervical and mediastinal adenopathy with left chest wall metastases. (Figure 2(a)). Testing for EGFR and ALK gene rearrangement was re-
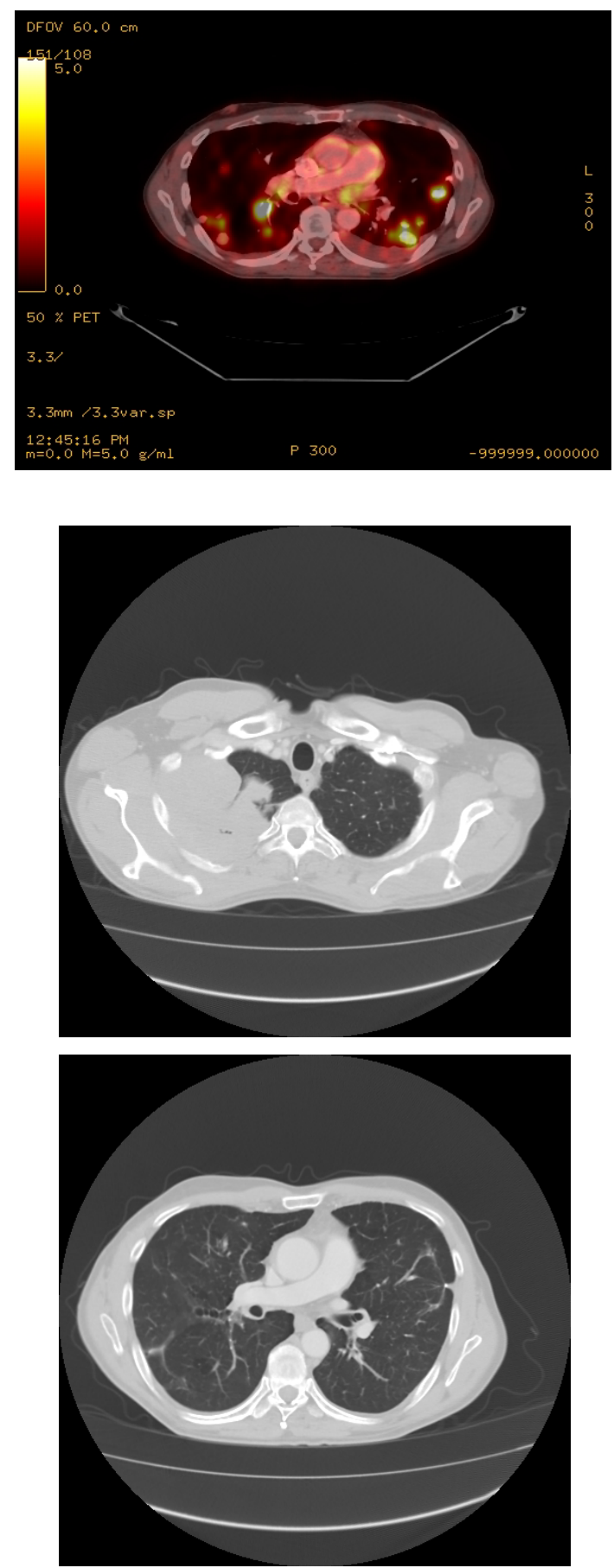

(b)

Figure 2. (a) Initial PET CT of chest, abdomen and pelvis revealed multiple FDG avid nodules in the right and left lung with a large intensely FDG avid mass in the right upper lobe, significant cervical and mediastinal adenopathy with left chest wall metastases; (b) CT scan three months after treatment started showed initially suspected mixed response with interval increase in the size of the right upper lobe mass measuring approximately $9.3 \times 9.1 \times 4.4 \mathrm{~cm}$, previously $6.1 \times 6.4 \times 4.4 \mathrm{~cm}$. There was near complete resolution of previously seen bilateral pulmonary nodules as well as the size of supraclavicular and mediastinal lymph nodes. 
quested revealing positive ALK gene translocation and Crizotinib $250 \mathrm{mg}$ twice daily was prescribed.

Three months later, repeat CT scan showed initially suspected mixed response with interval increase in the size of the right upper lobe mass measuring approximately $9.3 \times 9.1 \times 4.4 \mathrm{~cm}$, previously $6.1 \times 6.4 \times 4.4 \mathrm{~cm}$. There was near complete resolution of previously seen bilateral pulmonary nodules as well as the size of supraclavicular and mediastinal lymph nodes (Figure 2(b)). Due to discordant tumor response to therapy, biopsy of the right upper lobe mass was performed by interventional radiology for histology and ALK gene testing with concern that the lesion may be negative for the ALK gene translocation. Interestingly, pathology and immunohistochemical staining of the mass revealed a second primary consistent with small cell lung carcinoma (SCLC) (Figure 3). The patient is currently receiving systemic chemotherapy with carboplatin and etoposide for limited stage small cell lung cancer. Crizotinib therapy will be resumed after completion of therapy for SCLC.

\section{DISCUSSION}

For most patients with NSCLC, cytotoxic systemic chemotherapy remains first line treatment [3]. However, over the last five years novel therapeutic strategies for a subset of patients have emerged. NSCLC patients with activating mutations in the receptor tyrosine kinase epidermal growth factor receptor (EGFR) have sensitivity to EGFR tyrosine kinase inhibitors (TKI), specifically gefitinib or erlotinib [4]. In the landmark IPASS study, gefitinib was proven to be superior to cytotoxic chemotherapy for patients with known EGFR mutations in advanced, untreated disease [5].

In 2007, Soda et al. identified the newest molecular driver mutation in NSCLC susceptible to targeted therapy, the EML4-ALK fusion gene [6]. A small inversion within chromosome 2p, expresses a chimeric tyrosine ki-

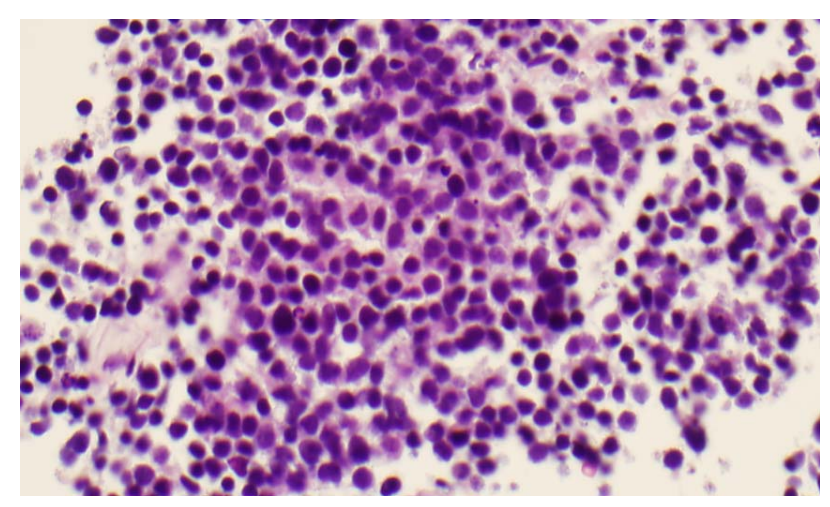

Figure 3. Pathology and immunohistochemical staining of the right upper lobe mass revealed a second primary consistent with small cell lung carcinoma (SCLC). nase in which the N-terminal portion of the echinoderm microtubule-associated protein-like 4 (EML4) is fused to the intracellular kinase domain of anaplastic lymphoma kinase (ALK) [7]. Only 2\% - 7\% of NSCLC harbor the EML4-ALK fusion [8]. Tumor cells with this rearrangement are susceptible to ALK kinase inhibitors facilitating downstream suppression of survival signaling pathways [9]. Crizotinib, a small molecule ALK tyrosine kinase inhibitor, is the latest targeted therapeutic treatment for NSCLC. In 2009, the results of crizotinib therapy in the first 19 patients with ALK-rearranged NSCLC were presented [10]. Overall response rate was found to be $53 \%$ (ten responders out of the first 19 patients) and five additional patients with stable disease resulting in a disease control (overall response rate + stable disease) rate of $79 \%$. Another early phase clinical trial reported that crizotinib therapy resulted in tumor shrinkage or stable disease in 82 patients with advanced NSCLC in 2010 [11]. Overall response rate was $57 \%$ and $33 \%$ of patients were noted to have stable disease resulting in a disease control rate of 90\%. Finally, in 2011 treatment efficacy data from two clinical trials of 255 ALK-rearranged NSCLC patients resulted in the conditional approval by the FDA of crizotinib [12]. Results included a response rate of $61 \%$ with an estimated progression free survival of nearly 10 months.

Shaw et al. reported that patients with EML4-ALK mutant tumors were significantly younger, more likely to be male, light or never smokers and have histology of adenocarcinoma with signet ring cell subtype [13]. However, subsequent studies have shown that EML4-ALK has been variably identified in both smokers and nonsmokers [14].

Synchronous multiple primary lung cancer (SMPLC) is defined by Martini et al. as lung cancers simultaneously diagnosed of either different histological types or two tumors of the same histological type in separate lobes without involvement of common lymphatics [15]. Wang et al. reported in 1999 on the potential etiology of SMPLC being secondary to increased tobacco exposure resulting in mutations in tumor suppressor genes [16]. An accurate diagnosis is crucial as prognosis and treatment options vary between metastatic disease and multiple primary lung cancers. The incidence of SMPLC is rare and the literature reports a range from $3.7 \%$ to $8.0 \%$ [17].

In conclusion, our case highlights the clinical relevance of the phenomena that is SMPLC. First, it reminds us of the necessity to avoid the misconception of a "mixed or partial response" when treating suspected metastatic lung disease as this directly impacts patient treatment and prognosis. Secondly, it suggests that the low reported incidence of SMPLC may be secondary to a low clinical suspicion in practice. 


\section{REFERENCES}

[1] Jemal, A., Siegel, R., Ward, E., et al. (2008) Cancer statistics, 2008. CA: A Cancer Journal for Clinicians, 58, 71-96. doi:10.3322/CA.2007.0010

[2] Soda, M., Choi, Y.L., Enomoto, M., et al. (2007) Identification of the transforming EML4-ALK fusion gene in non-small cell lung cancer. Nature, 448, 561-566. doi:10.1038/nature05945

[3] Schiller, J.H., Harrington, D., Belani, C.P., et al. (2002) Comparison of four chemotherapy regimens for advanced non-small cell lung cancer. The New England Journal of Medicine, 346, 92-98. doi:10.1056/NEJMoa011954

[4] Lynch, T.J., Bell, D.W., Sordella, R., et al. (2004) Activating mutations in the epidermal growth factor receptor underlying responsiveness of non-small cell lung cancerto gefitinib. The New England Journal of Medicine, 350, 2129-2139. doi:10.1056/NEJMoa040938

[5] Mok, T., Wu, Y.-L., Thongprasert, S., et al. (2008) Phase III, randomized, open-label, first line study of gefitinib vs carboplatin/paclitaxel in clinically selected patients with advanced non-small cell lung cancer (IPASS). 33rd European Society for Medical Oncology Congress, Stockholm, 12-16 September 2008.

[6] Soda, M., Choi, Y.L., Enomoto, M., et al. (2007) Identification of the transforming EML4-ALK fusion gene in non-small cell lung cancer. Nature, 448, 561-566. doi:10.1038/nature05945

[7] Chiarle, R., Voena, C., Ambrogio, C., et al. (2008) The anaplastic lymphoma kinase in the pathogenesis of cancer. Nature Reviews Cancer, 8, 11-23. doi:10.1038/nrc2291

[8] Rikova, K., Guo, A., Zeng, Q., et al. (2007) Global survey of phosphotyrosine signaling identifies oncogenic kinases in lung cancer. Cell, 131, 1190-1203. doi:10.1016/j.cell.2007.11.025

[9] Koivunen, J.P., Mermel, C., Zejnullahu, K., et al. (2008) EML4-ALK fusion gene and efficacy of an ALK kinase inhibitor in lung cancer. Clinical Cancer Research, 14,
4275-4283. doi:10.1158/1078-0432.CCR-08-0168

[10] Kwak, E.L., Camidge, D.R., Clark, J., et al. (2009) Clinical activity observed in a phase I dose escalation trial of an oral c-met and ALK inhibitor, PF-02341066. Journal of Clinical Oncology, 27, 3509

[11] Kwak, E.L., Bang, Y.-J., Camidge, D.R., et al. (2010) Anaplastic lymphoma kinase inhibition in non-small-cell lung cancer. The New England Journal of Medicine, 29, e443-e445

[12] Camidge, D.R., Bang, Y., Kwak, E.L., et al. (2011) Progression-free survival (PFS) from a phase I study of crizotinib (PF-02341066) in patients with ALK-positive nonsmall cell lung cancer (NSCLC). Journal of Clinical Oncology, 29, 2501.

[13] Shaw, A.T., Yeap, B.Y., Kenudson-Mino, M., et al. (2009) Clinical features and outcome of patients with non-small cell lung cancer who harbor EML4-ALK. Journal of Clinical Oncology, 27, 4247-4253. doi:10.1200/JCO.2009.22.6993

[14] Shinmura, K., Kageyama, S., Tao, H., et al. (2008) EML4 ALK fusion transcripts, but no NPM, TPM3, CLTC, ATIC, or TGF-ALK fusion transcripts, in non-small cell lung carcinoma. Lung Cancer, 61, 163-169. doi:10.1016/j.lungcan.2007.12.013

[15] Martini, N. and Melamed, M.R. (1975) Multiple primary lung cancer. The Journal of Thoracic and Cardiovascular Surgery, 70, 606-612

[16] Wang, X., Christiani, D.C., Mark, E.J., et al. (1999) Carcinogen exposure, p53 alteration, and K-ras mutation in synchronous multiple primary lung carcinoma. Cancer, 85, 1734-1739. doi:10.1002/(SICI)1097-0142(19990415)85:8<1734::AI D-CNCR13>3.0.CO;2-1

[17] Rostad, H., Strand, T.E., Naalsund, A. and Norstein, J. (2008) Resected synchronous primary malignant lung tumors: A population-based study. The Annals of Thoracic Surgery, 85, 204-209. doi:10.1016/j.athoracsur.2007.07.091 